\title{
DE LA CALLE A LA MESA. ACCIONES DE PROTESTA $Y$ OPORTUNIDADES POLIITICAS EN EL GOBIERNO DE LUIS GUILLERMO SOLÍS (2014-2018)
}

\section{PROTEST ACTIONS AND POLITICAL OPPORTUNITIES IN THE GOVERNMENT OF LUIS GUILLERMO SOLÍS (2014-2018)}

\author{
Alejandro Alvarado Alcázar* \\ Gloriana Martínez Sánchez ${ }^{* *}$
}

RESUMEN

El artículo muestra los resultados de un trabajo sistemático de monitoreo de la protesta social en Costa Rica durante el cuatrienio de gobierno de Luis Guillermo Solís (mayo 2014mayo 2018), mediante la metodología de Análisis de Eventos de Protesta (AEP). Para esto, el documento se estructura en dos partes. La primera parte se enfoca en describir y caracterizar las tendencias generales de la protesta durante el período de estudio, en términos de los repertorios de acción, los actores demandantes, las demandas y los actores demandados. De esta parte, se concluye que las acciones de protesta decrecieron año con año y que las estrategias y rutinas de acción fueron diferenciadas según los actores demandantes. La segunda parte propone como hipótesis de trabajo a futuro, explorar la relación entre la reducción de las acciones de protesta y las oportunidades políticas abiertas por el gobierno de Solís.

PALABRAS CLAVE: MOVIMIENTOS SOCIALES * ACCIÓN COLECTIVA * PROTESTA SOCIAL * OPORTUNIDADES POLÍTICAS

$1 \quad$ El presente trabajo es parte de los resultados de la actividad de investigación "Seguimiento de las acciones colectiva en Costa Rica" inscrita en el Instituto de Investigaciones Sociales de la Universidad de Costa Rica (IIS-UCR).

* Instituto de Investigaciones Sociales (IIS) y Escuela de Sociología de la Universidad de Costa Rica, Costa Rica. omar.alvaradoalcazar@ucr.ac.cr

** Instituto de Investigaciones Sociales (IIS), Centro de Investigaciones y Estudios Políticos (CIEP) y Escuela de Ciencias Políticas de la Universidad de Costa Rica, Costa Rica.

gloriana.martinezsanchez@ucr.ac.cr 


\section{ABSTRACT}

This paper shows the results of a systematic work of monitoring social protest in Costa Rica during the four-year term of the government of Luis Guillermo Solís (May 2014-May 2018), using the Protest Event Analysis (AEP) methodology. The document is structured in two parts. The first focuses on describing and characterizing the general tendencies of the protest during the study period, it looks at the protest repertoires, the demanding actors, the demands and the demanded actors. From this part, it is concluded that the protest actions decreased year after year and that the strategies and action routines were differentiated according to the demands. The second part proposes exploring the relationship between the reduction of protest actions and the political opportunities opened by the Solis government as a working hypothesis for future research. KEYWORDS: SOCIAL MOVEMENTS * COLLECTIVE ACTION * SOCIAL PROTEST * POLITICAL
OPPORTUNITIES

\section{INTRODUCCIÓN}

El domingo 6 de abril de 2014, Luis Guillermo Solís, candidato del Partido Acción Ciudadana (PAC), resultó electo como presidente de Costa Rica, tras triunfar en segunda ronda con un apoyo electoral de más de 1,3 millones de votos (78\%). El triunfo de Solís y su partido representó el fin de un ciclo electoral de más de 30 años, durante el cual los dos partidos políticos tradicionales, Liberación Nacional (PLN) y Unidad Social Cristiana (PUSC), intercambiaron triunfos electorales, siendo esta la primera ocasión en que un partido diferente a los dos mencionados, se hacía con el control del poder ejecutivo.

Diferentes estudios de opinión pública sobre democracia y elecciones en Costa Rica constataban que, en la última década, la ciudadanía costarricense mostraba una lectura pesimista de la política y de la gestión gubernamental (Raventós, Fournier, Ramírez, Gutiérrez y García, 2005; Cascante y Pignataro, 2018). Dicho malestar con la política electoral y el sostenido descontento con las estructuras partidarias generó profundos cambios en las formas de identificación política $y$ de participación electoral, transformando las conductas ciudadanas y electorales (Raventós, Fournier, Fernández y Alfaro, 2012; Cascante y Pignataro, 2018), con lo cual el resultado electoral de 2014 representó una manifestación más.
Estas transformaciones, no siempre traducidas en protesta social, junto con el considerable desgaste de las estructuras partidarias tradicionales, fueron elementos determinantes para el triunfo electoral de Solís, quien generó un cúmulo de expectativas de cambio entre la ciudadanía, las cuales se fueron desinflando con el pasar de los meses (CIEP, 2018).

Las mediciones señalan que, durante sus primeros meses de gestión, el gobierno fue bien calificado y la población tenía consideraciones positivas sobre el futuro político. No obstante, pasado su "período de prueba", el apoyo fue decreciendo sostenidamente $y$ las consideraciones se tornaron negativas, llegando a tener puntajes de descontento social similares a los de su antecesora, Laura Chinchilla (2010-2014) (CIEP, 2014, 2015, 2016 y 2018).

Pese a lo anterior, tal como se discute en este trabajo, los datos referidos a las acciones de protesta registradas durante el cuatrienio de gobierno de Solís muestran dos hechos que, en principio, contrastan con esto último: 1) la tendencia decreciente de las acciones de protesta y 2) la predominancia de los repertorios institucionales sobre los no-institucionales.

De acuerdo con la metodología de Análisis de Eventos de Protesta (AEP), el presente artículo se centra en describir y caracterizar las tendencias generales de la protesta durante el período de estudio, identificando los patrones predominantes en términos de los repertorios de acción, los actores demandantes, 
las demandas y los actores demandados. Posteriormente, la segunda parte propone una hipótesis de trabajo a futuro para interpretar por qué se redujo la protesta durante el gobierno de Solís. Concretamente, el artículo plantea explorar el tipo de manejo dado por el gobierno a la protesta $y$ a los reclamos de los actores demandantes, como una línea de interpretación para entender dicho fenómeno. Para esto, se propone una discusión que recupera elementos de la teoría de los movimientos sociales relacionados con el papel de las oportunidades políticas para explicar la dinámica de la protesta social en una coyuntura determinada.

Para el desarrollo de lo mencionado, el artículo se estructura en cuatro secciones. En primer lugar, discute sobre la categoría de protesta, en un sentido teórico y metodológico. En segundo lugar, muestra las tendencias generales de la protesta durante el cuatrienio. En tercer lugar, plantea la discusión teórica alrededor de la Estructura de Oportunidades Políticas (EOP). En cuarto lugar, plantea como interrogante si la estructura de las oportunidades políticas durante el gobierno de Luis Guillermo Solís, se constituye como un factor que contribuye a explicar la tendencia a la reducción de las acciones de protesta durante el periodo.

\section{EL ANÁLISIS DE EVENTOS DE PROTESTA (AEP): ELEMENTOS TEÓRICOS Y METODOLÓGICOS}

\section{UNA DEFINICIÓN DE PARTIDA}

Durante la década de 1990, la categoría de protesta social se tornó de uso común entre una serie de estudios latinoamericanos dedicados a entender la dinámica de la conflictualidad social (Svampa, 2009). Mediante dicha categoría se habilitó la reconstrucción de los procesos de lucha social desde la identificación de los repertorios de acción, los actores demandantes, las demandas, los actores demandados y otra serie de categorías analíticas. También, se tornó posible relacionar la dinámica de la protesta con los procesos de cambio social, político y económico a escala nacional y transnacional (Schuster et ál., 2006; Retamozo y Trujillo, 2018); y, de la misma manera, entender la dinámica de continuidad $y$ discontinuidad entre la llamada política institucional y la política no-institucional, siendo esta última el terreno clásico de la protesta social.

La metodología de AEP, muy común en los estudios sobre la protesta social, ofrece una entrada analítica sumamente útil para la reconstrucción de este fenómeno, mediante la consulta de fuentes hemerográficas (prensa escrita). El AEP consiste en el registro sistemático de eventos de protesta y su procesamiento atendiendo a elementos que se consideran fundamentales en la estructuración de una acción de protesta, tales como, los repertorios de acción, los actores demandantes, las demandas y los actores demandados.

El presente artículo sigue precisamente la metodología de AEP para la reconstrucción de la dinámica de la protesta social en Costa Rica, centrándose en la identificación de una serie de elementos típicos de lo que se constituye como una acción de protesta.

En primer lugar, se considera que toda protesta tiene un carácter colectivo, es decir, que se trata de acciones colectivas concertadas. En segundo lugar, que desarrolla uno o más repertorios, entendidos como rutinas de acción mediante las cuales, los actores plantean su disconformidad con determinado tema. En muchos trabajos que siguen la metodología de AEP, solo se consideran las acciones de protesta que ocurren en espacios públicos (Cadena-Roa, 2016), también conocidas como acciones contenciosas o no-institucionales; no obstante, en el caso de este trabajo, también se registran acciones de carácter institucional, tales como, las denuncias ante entidades, atendiendo a características propias de la cultura política costarricense que hacen que muchos de los descontentos de la ciudadanía se procesen primero mediante canales institucionales. Adicionalmente, se considera que la reconstrucción de las acciones institucionales ofrece una mejor mirada sobre la dinámica de la protesta, posibilitando la identificación de los "ciclos" de la acción de protesta, la cual en muchos casos, se desarrolla no solo en la "calle".

En tercer lugar, se debe registrar un actor demandante, es decir, un actor colectivo 
que plantea un reclamo particular. En cuarto lugar, toda acción de protesta es portadora de una demanda. Finalmente, para que se considere una acción de protesta es necesario identificar un actor demandado, es decir, el actor-estatal o no-estatal contra quien se dirige la demanda.

De esta manera, en este trabajo la acción de protesta se entiende como una forma de acción de carácter colectivo desarrollada mediante el recurso a repertorios de acción, tanto institucionales como no-institucionales, por un actor demandante con el fin de plantear un reclamo (demanda) ante un actor demandado (Alvarado y Martínez, 2018). A partir de esta definición, es que mediante la base de datos PROTESTAS (protestas.iis.ucr.ac.cr) se mantiene un monitoreo constante de las acciones de protesta reportadas en las ediciones digitales de cuatro medios de comunicación escrita ${ }^{2}$ : La Nación, La Extra, CR Hoy y Socialismo Hoy.

Para el procesamiento de la información mediante la base de datos PROTESTAS, se definieron un conjunto de categorías primarias: 1 ) repertorio de protesta; 2 ) actor demandante; 3 ) demanda; y 4) actor demandado, las cuales son consistentes con lo planteado por la metodología de $\mathrm{AEP}^{3}$.

La categoría de repertorio de protesta tiene una reconocida trayectoria en el estudio de la acción colectiva. Para Tilly (2002), uno de los autores más destacados en el análisis de los repertorios de acción colectiva, esta

2 La selección de las fuentes consideró la cobertura territorial de los medios, pues interesaba que estos cubrieran el territorio nacional y no solamente la escala local o regional. También se consideró como un criterio la periodicidad de las publicaciones, prefiriendo recurrir a medios de publicación diaria.

3 Cabe señalar que estas no son todas las categorías usadas en PROTESTAS, sino las que definen que una determinada acción colectiva se considere como una acción colectiva de protesta. Además de las categorías mencionadas, la base de datos también considera la delimitación temporal y espacial de las protestas, los tipos de respuesta y los tipos de represión de los actores demandados, entre otras (ver Guía Metodológica en https://protestas.iis.ucr. ac.cr/publicaciones/173). categoría se refiere a un conjunto limitado de rutinas conocidas de acción, las cuales están inscritas culturalmente en la experiencia de los actores de la protesta, por lo que en cada momento histórico estos manejan una cantidad limitada de formas de protestar (pp. 8-9). Este autor sostiene que los repertorios deben estudiarse en términos de las interacciones que generan entre los actores de la contienda política, pues “...en el marco de un repertorio establecido, cada rutina consiste en una interacción entre dos o más partes. Los repertorios corresponden a un conjunto de actores [colectivos] confrontados, no a actores individuales" (2002, p. 11).

Para efectos de este trabajo, se tomó la decisión de distinguir entre repertorios institucionales y no-institucionales, definidos según el lugar (espacio físico y político) donde ocurren y su carácter contencioso. Los repertorios institucionales se han definido como formatos de acción que transcurren en espacios y mecanismos institucionales $y$ formales, por lo que este tipo de repertorio no necesariamente ocurre en el espacio público y su capacidad de transgresión e impacto inmediato es reducido, ya que recurren a las dinámicas e instancias formales del sistema político para procesar las demandas $y$ los reclamos de los actores colectivos ${ }^{4}$.

En el caso de los repertorios no-institucionales, estos son los que la teoría típicamente define como contenciosos, pues ocurren en el espacio público, popularmente nombrado como la "calle" . Autores como Tarrow y el mismo Tilly, han señalado que los repertorios de carácter contencioso son fundamentales en la dinámica de la acción colectiva, pues contribuyen a integrar a los individuos en esta (Tarrow, 1997), y constituyen parte de las rutinas de los movimientos sociales. De la misma manera, los repertorios, particularmente los no-institucionales, suponen una transgresión

4 Incluye los repertorios: declaración pública, reunión ante autoridades, denuncia ante entidades, ciberacciones.

5 Incluye los repertorios: reunión, huelga de hambre, concentración, marcha, paro, huelga, bloqueo $y$ actos sobre la propiedad. 
en el espacio público que trastoca la cotidianeidad de quienes los desarrollan, de los actores demandados $y$ de terceros.

Ahora, si bien es fundamental destacar que tradicionalmente las acciones de protesta hacen referencia a formas no-institucionales de actuar, lo cierto es que entre estas formas $y$ las institucionales, generalmente se mantiene una relación de continuo, tanto en términos de la dinámica entre los actores, donde normalmente el demandado es un actor institucional, como en función de los repertorios de protesta. Esto configura lo que diferentes autores llaman como la dinámica de la contienda política (McAdam, Tarrow y Tilly, 2005), es decir, la interacción que se establece entre diferentes actores, tanto institucionales como no-institucionales, en el marco de un sistema político determinado.

En relación con la categoría de actor demandante, esta se refiere a los actores colectivos protagónicos de las acciones de protesta, es decir, quienes la desarrollan, la sostienen y generan recursos políticos para hacerla posible. Tal como se señaló, el tipo de actor que interesa particularmente es el colectivo o el que refiere a una colectividad, no el individual en sí mismo. Esto permite la inclusión de diferentes tipos de actores colectivos ${ }^{6}$, estén formal o informalmente organizados. En este sentido, los actores demandantes pueden ser de distinto tipo y con diferentes recursos, pero la mayoría comparte el hecho de que la acción de protesta contenciosa es un recurso recurrente para plantear sus demandas frente a otros actores con mayores recursos (Tarrow, 1997).

$6 \quad$ En el caso de la base de datos PROTESTAS se cuenta con 13 tipos de actores demandantes: 1) trabajadores y trabajadoras; 2) empresarios y empresarias; 3) campesinos, campesinas e indígenas; 4) pescadores $y$ pescadoras; 5) estudiantes, madres $y$ padres; 6) vecinas $y$ vecinos; 7) ciudadanos $y$ ciudadanas; 8) de derechos humanos; 9) de género; 10) de medio ambiente; 11) de vivienda; 12) de usuarios y usuarias; $y, 13)$ de profesionales. Además, para los tipos 1, 2, 3, 4, 5, 8 y 9 se han generado una serie de subtipos para una identificación más precisa en el interior de cada categoría (ver Guía Metodológica en https://protestas.iis.ucr.ac.cr/ publicaciones/173).
La categoría de demanda hace referencia a lo que se encuentra en disputa, es decir, lo que está en juego. Las demandas refieren a diferentes cuestiones económicas, políticas, culturales, materiales e inmateriales. Entre los actores se establece una interacción en torno a una demanda, la cual configura el terreno de conflicto entre estos, por lo tanto esta categoría es central para el análisis de la conflictualidad, ya que cuando un actor plantea una demanda, está posicionando públicamente un conflicto político en torno a cómo se distribuye un determinado recurso en una sociedad particular ${ }^{7}$.

Por su parte, en este trabajo se considera que el actor demandado, es también actor protagónico, pero en el sentido contrario, es el actor contra el cual se plantea la acción de protesta $y$ en algunos casos de quien se espera una solución a la demanda planteada. El actor demandado puede ser de distinto tipo, tanto institucional como no-institucional; no obstante, en sociedades como las latinoamericanas, el Estado en sus diferentes escalas y formas institucionales, es una constante en términos de las demandas ${ }^{8}$.

Al tomar en cuenta las categorías descritas anteriormente, se considera la protesta social como un terreno complejo de acción definido por la dinámica entre actores confrontados en torno a una demanda concreta. Dicha dinámica marca la relacionalidad interna, en la cual los actores se insertan con determinados

$7 \quad$ Igualmente, la base de datos cuenta con 13 tipos de demandas y sus correspondientes subtipos, las cuales consideran desde reclamos relacionados con el trabajo y las condiciones laborales hasta aquellos referidos a derechos específicos o la gestión pública (ver Guía Metodológica en https://protestas.iis.ucr.ac.cr/publicaciones/173).

8 La lista de actores demandados incluida en PROTESTAS suma un total de 22. Esta incluye a diferentes actores estatales, clasificados según poder de la República (legislativo, ejecutivo, judicial) y/o escala político-territorial (nacional, local). También considera a instituciones autónomas, tales como, la Caja Costarricense de Seguro Social (CCss), el Instituto Costarricense de Electricidad (ICE) o las universidades públicas. Las empresas privadas también se incluyen (ver Guía Metodológica en https://protestas.iis.ucr.ac.cr/publicaciones/173). 
recursos políticos, económicos, culturales, etc., que definen, en buena medida, sus márgenes de acción. De la misma manera, los actores, particularmente, los demandantes, desarrollan su acción inscritos en un entorno político que ofrece mayores o menores oportunidades para el desarrollo de sus acciones y para la consecución de sus demandas, condicionando, en diferentes grados, la dinámica de la protesta social.

En la sección siguiente, se muestran los datos de la protesta social registrados para el cuatrienio de gobierno de Luis Guillermo Solís, haciendo énfasis en los repertorios de acción y los actores demandantes.

\section{LA PROTESTA SOCIAL EN EL GOBIERNO DE LUIS GUILLERMO SOLÍS}

Entre el 8 de mayo de 2014 y el 7 de mayo de 2018, período en el que se desarrolló el mandato presidencial de Luis Guilllermo Solís, se registraron en la base de datos PROTESTAS, un total de 2475 acciones, según las fuentes consultadas ${ }^{9}$. Tal como se muestra en el gráfico 1, el primer año de gobierno ( 8 de mayo de 2014-7 de mayo de 2015) fue el de mayor cantidad de protestas en toda la serie de tiempo, concentrándose el 33,3\% (832 protestas) de los registros. Luego de esto, en los períodos posteriores, la tendencia marcó un decrecimiento constante de la protesta, siendo el cuarto y último año de gobierno, el de menor cantidad de acciones (18,9\% / 469 protestas) (Alvarado y Martínez, 2018).

\section{GRÁFICO 1 \\ ACCIONES DE PROTESTA SEGÚN PERIODO \\ COSTA RICA, 07/05/2014 AL 07/05/2018 \\ (DISTRIBUCIONES PORCENTUALES)}

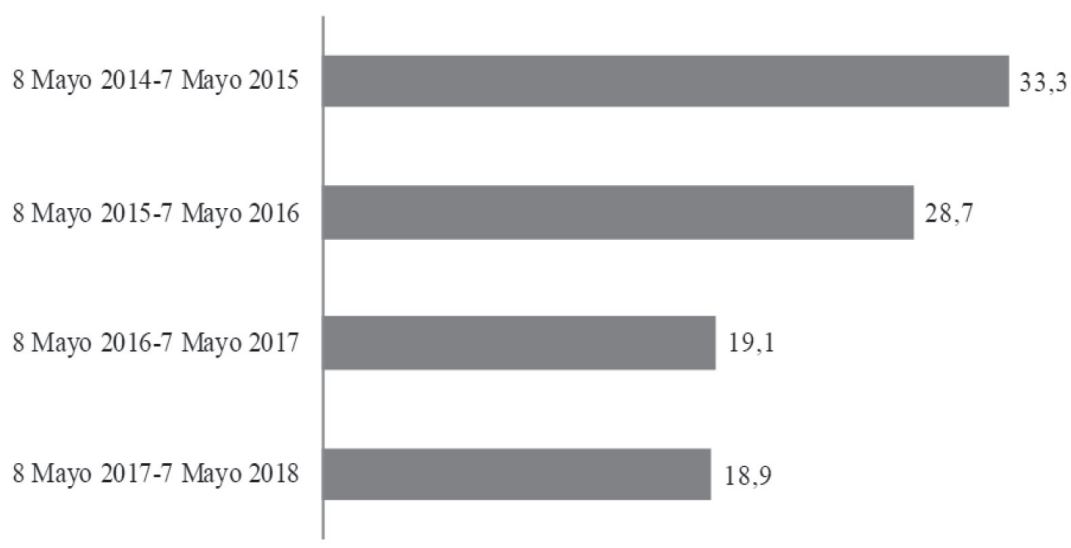

Fuente: $\quad$ PROTESTAS, base de datos colectivas, IIS-UCR, 2014-2018.

9

Si bien, es necesario considerar que la unidad de análisis temporal usada en este trabajo comprende el mandato presidencial de Luis Guillermo Solís, no necesariamente todas las acciones de protesta registradas durante este período se le pueden atribuir a Solís y a su gobierno. Esta lectura no distaría de una interpretación de los regímenes políticos como presidencialistas, en la cual la figura que ostenta la presidencia concentraría prácticamente toda la capacidad de decisión sobre lo que sucede en un Estado. En este caso, se ha sopesado este elemento, pero se ha decidido mantener este encuadre temporal como una forma de acercarse a la lectura de la coyuntura. Otros análisis podrán introducir otros elementos. 
Esta tendencia decreciente de la protesta resulta contrastante con lo ocurrido durante el gobierno de Laura Chinchilla (2010-2014), pues otras mediciones de las acciones de protesta en Costa Rica (Guillén, 2016) han señalado que dicho período fue el de mayor conflictualidad social reciente en el país, siendo el año 2012 el que presenta el mayor pico en la cantidad de protestas de ese gobierno.

En cuanto a la dinámica de los repertorios de protesta durante el cuatrienio de
Solís, se muestra la consolidación de un conjunto de rutinas, tanto de carácter institucional como no-institucional, que mantienen una estrecha relación con la cultura política costarricense, en la cual los mecanismos institucionalizados de la democracia procedimental continuan siendo considerados como la mejor manera de procesar los diferendos sociales y políticos (Álvarez, 2011 y 2015) ${ }^{10}$. En el gráfico 2, se muestra los repertorios más recurrentes durante el cuatrienio.

\section{GRÁFICO 2 \\ ACCIONES DE PROTESTA SEGÚN REPERTORIO DE ACCIÓN \\ COSTA RICA, 2014-2018 \\ (DISTRIBUCIONES PORCENTUALES)}

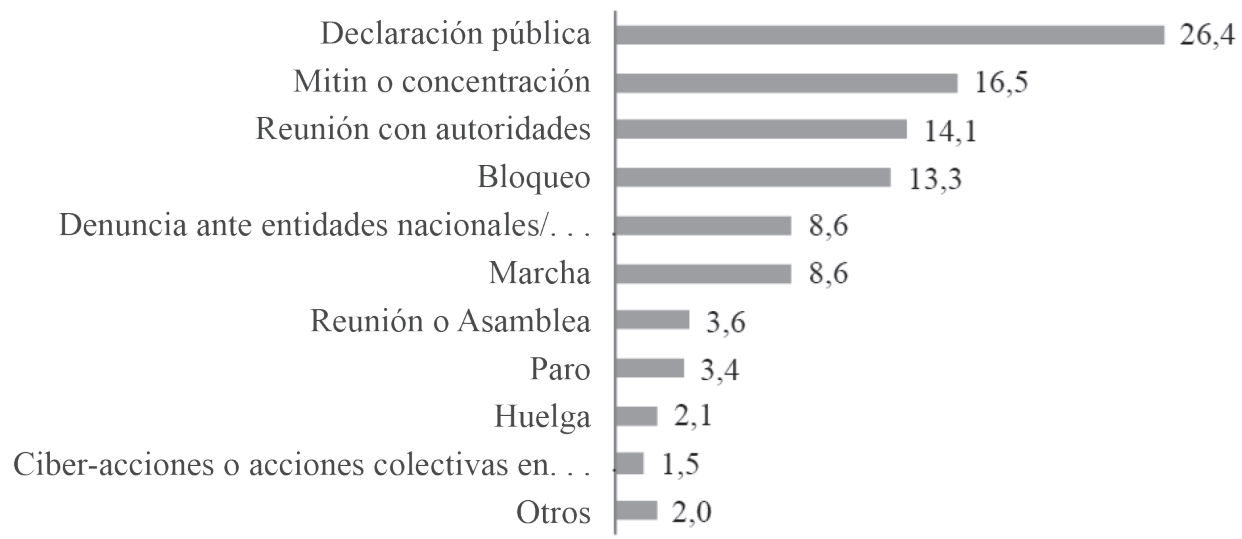

Fuente: PROTESTAS, base de datos colectivas, IIS-UCR, 2014-2018.

$10 \quad$ Estudios de opinión pública señalan que en Costa Rica, la protesta social contenciosa es considerada por la ciudadanía como un método poco efectivo y poco usado para solucionar problemas, mientras que las actitudes políticas relacionadas con la obediencia mantienen mayor importancia entre la ciudadanía que las referidas a la participación de carácter no-institucional (Cascante y Pignataro, 2018). 
Respecto a los repertorios de protesta, se identificó una tendencia a la combinación de diferentes rutinas de acción entre los actores demandantes para plantear sus reclamos. Es decir, un actor colectivo en el marco de una misma demanda y en una misma coyuntura, tiende a combinar diferentes repertorios, tal como una marcha, un comunicado de prensa y una denuncia ante una entidad, entre otras.
A partir de esta combinación de repertorios de protesta, se constata que tanto las acciones de carácter institucional como noinstitucional fueron sistemáticamente usadas durante el período (gráfico 3), pero con predominancia de las primeras que fueron usadas en el $54,5 \%$ de las protestas registradas. Por el contrario, los repertorios de tipo no-institucional, es decir, los formatos típicamente contenciosos, se registraron en 45,1\% de las acciones.

\section{GRÁFICO 3 \\ ACCIONES DE PROTESTA SEGÚN CAMPO DE ACCIÓN \\ COSTA RICA, 2014-2018 \\ (DISTRIBUCIONES PORCENTUALES)}

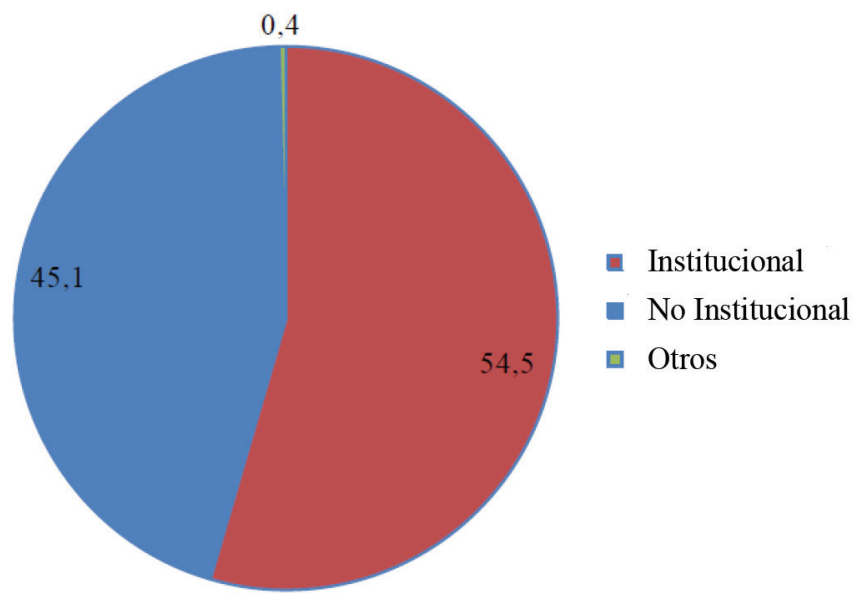

Fuente: $\quad$ PROTESTAS, base de datos colectivas, IIS-UCR, 2014-2018.

Esta tendencia a la combinación de repertorios institucionales y no-institucionales, con predominio de los primeros, es consistente con lo planteado por Calderón (2012), según el cual en países donde la institucionalidad muestra una mayor capacidad para procesar las demandas de la población, se registra, en términos generales, una menor cantidad de conflictos sociales con una menor radicalidad. De hecho, este autor sostiene que los patrones históricos de la cultura política costarricense y su institucionalidad generan que los conflictos se procesen tendencialmente mediante el diálogo institucionalizado y la negociación formal.

La relación entre repertorios institucionales y no-institucionales mostrada en el gráfico 3, plantea que, durante el gobierno de Solís, los mecanismos propios de la institucionalidad formal fueron sistemáticamente recurridos entre los actores demandantes para reclamar a los actores demandados, particularmente a los estatales. Esto no implica un distanciamiento total de la calle como espacio de acción política, sino una redefinición de la dinámica entre los diferentes tipos de repertorio, donde los 
formatos institucionales cobraron una mayor centralidad para determinados actores.

En este sentido, es posible plantear que la dinámica entre repertorios institucionales y no-institucionales toma la forma de un continuo, pues, en general, los actores combinan diferentes formatos de protesta en el desarrollo de sus campañas. Dicha combinación estaría sujeta a la dinámica más general de interacción entre actores demandantes $y$ actores demandados, de tal manera que el tipo de repertorio escogido para manifestar las demandas respondería a la estrategia y recursos de quienes protestan y a los tipos de respuesta que reciben de la institucionalidad.

Por su parte, durante el gobierno de Solís destacaron tres actores ${ }^{11}$ cuyas demandas articularon mayoritariamente la dinámica de la protesta: 1) la población trabajadora; 2) las madres, los padres y la población estudiantil, particularmente los de secundaria; y 3) las vecinas y los vecinos.

En el caso de la población trabajadora, estos fueron protagonistas en prácticamente 1 de cada 2 protestas registradas en el período. Sus reclamos fueron de contenido fundamentalmente gremial, entre los que destacan demandas relacionadas con la defensa de sus derechos laborales y salariales, así como, sus libertades sindicales. No obstante, este actor también protagonizó protestas referidas a nudos de conflicto de carácter general y nacional, tales como, los proyectos de ley sobre reforma fiscal, educación dual y empleo público, las transformaciones en los regímenes de pensiones y la denuncia de casos de corrupción.

Si se compara con otros actores de la protesta, los recursos políticos con los que cuentan los sindicatos son considerablemente mayores, lo cual les permite, en muchos casos, contar con más y mejores oportunidades políticas de cara a los gobiernos de turno. Este fue el caso durante el gobierno de Solís, período en el cual los sindicatos contaron con un mayor acceso a la institucionalidad (Guillén, 2016).

En el caso de la población trabajadora $y$, particularmente, de las organizaciones sindicales, es común que cuente con una mayor cantidad de recursos, en comparación con otros actores, lo que les posibilita generar acciones de protesta $y$, sobre todo, llamar la atención de las autoridades sin necesariamente hacer uso (de entrada) de repertorios de carácter noinstitucional, particularmente en coyunturas donde las oportunidades políticas parecen más abiertas para el diálogo con estos sectores organizados (Guillén, 2016).

De hecho, como se muestra en el gráfico 4, de un total de 1224 acciones en las que este sector fue el actor protagónico, en un $62,8 \%$ se hicieron mediante un repertorio institucional, mientras que solo en un $36,9 \%$ usaron un repertorio no-institucional.

11 Las personas trabajadoras del sector público y privado protagonizaron el 49,5\% de todas las acciones de protesta registradas en el período, la población estudiantil, las madres y los padres de familia sumaron un $11,2 \%$, las vecinas y los vecinos contabilizaron un 10,2\% (Alvarado y Martínez, 2018). 


\author{
GRÁFICO 4 \\ ACCIONES DE PROTESTA POR PARTE DE LA POBLACIÓN TRABAJADORA \\ SEGÚN CAMPO DE ACCIÓN \\ COSTA RICA, 2014-2018 \\ (DISTRIBUCIONES PORCENTUALES)
}

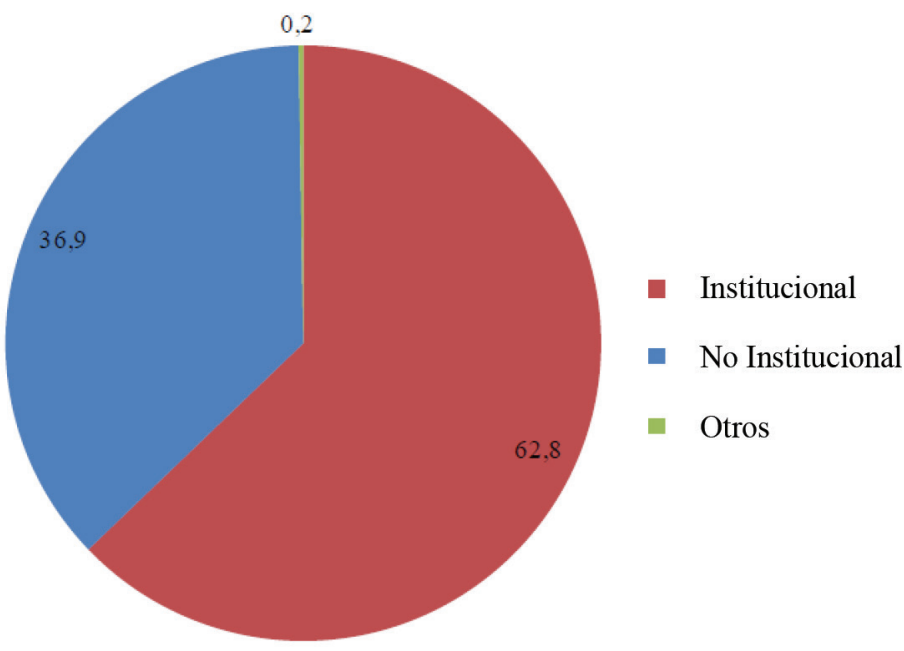

Fuente: $\quad$ PROTESTAS, base de datos colectivas, IIS-UCR, 2014-2018.

Lo anterior cuestiona la idea constantemente reproducida por actores hegemónicos, como las élites político-económicas y las empresas de comunicación, de que las personas trabajadoras, particularmente, las que laboran en el sector público, siempre recurren a la calle $y$ a repertorios como el paro o la huelga para plantear sus demandas. Por el contrario, los datos muestran que los sindicatos hicieron un uso predominante de los mecanismos institucionales y que la calle se transforma en recurso, sobre todo, cuando se enfrentan a entornos políticos cerrados.

En relación con los actores demandados por esta población, estos refieren a la dinámica entre este sector $y$ diferentes instancias institucionales. El poder ejecutivo (gobierno central) fue el actor más demandado, con un 42,9\% de todas las acciones registradas. Le siguieron las instituciones autónomas con un 19,0\% y el poder legislativo con un $10,4 \%$.

Estos datos muestran cómo el Estado y su entramado institucional mantienen un rol fundamental en la dinámica de la protesta, tanto interpelado en su carácter de generador de conflictos como de (potenciales) soluciones. Esto es particularmente cierto en el caso de las trabajadoras y los trabajadores públicos, pues el Estado toma una presencia multiforme en el caso de este sector. Se presenta bajo la forma de patrón, pero también como mediador en los conflictos, siendo el caso de ciertas autoridades e instituciones como el presidente de la República o el Ministerio de Trabajo (MTss).

Por su parte, una de las características centrales de las acciones de protesta de madres, padres $y$ estudiantes, segundo actor con más acciones registradas durante el período $(11,2 \%)$, fue el recurso frecuente a repertorios de tipo contencioso (no-institucionales), los cuales correspondieron a un $77,6 \%$ de todas sus acciones (gráfico 5). Es importante destacar que madres $y$ padres de familia $y$ estudiantes dirigieron fundamentalmente sus demandas contra el poder ejecutivo $(76,5 \%)$, particularmente, al Ministerio de Educación Pública (MEP). 

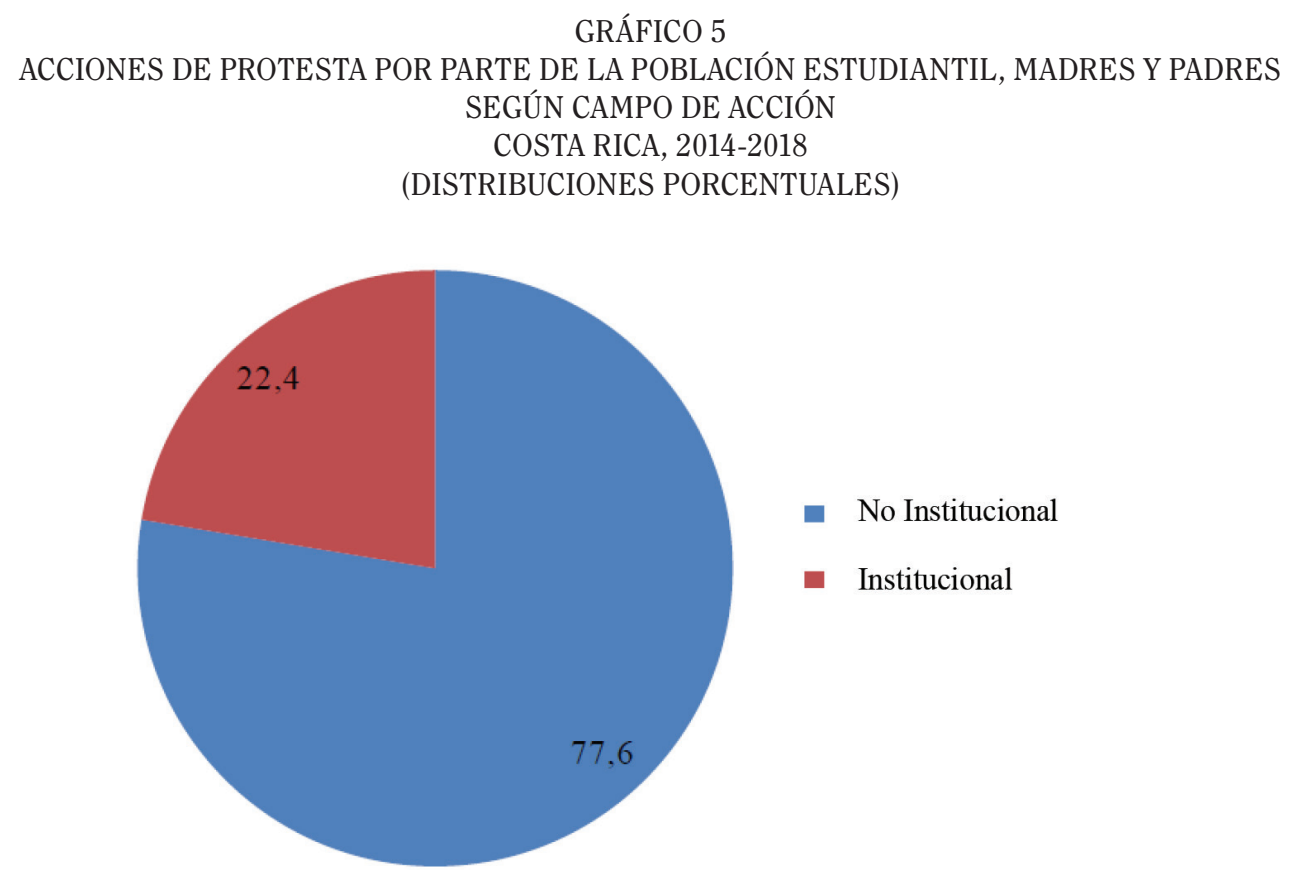

Fuente: $\quad$ PROTESTAS, base de datos colectivas, IIS-UCR, 2014-2018.

La distinción entre el uso de estos formatos de protesta responde en gran medida a los recursos con los que cuentan las madres, las padres y la población estudiantil para acceder a la institucionalidad y conseguir solución a sus demandas.

En cuanto a las demandas de este actor, se registraron las de tipo particular y de carácter local, relacionadas con descontentos con el funcionamiento de centros de educación, en donde solicitan la destitución de personas funcionarias y la mejora de infraestructuras; $y$ las de tipo general y de carácter nacional, relacionadas con el financiamiento de la educación superior pública y la eliminación de contenidos de educación. No obstante, es el primer tipo de demanda el que predomina en este sector, por lo cual el uso de formatos no-institucionales, que corresponde con los recursos con que cuentan estos actores, fue lo más usual. Particularmente, los cierres (bloqueos) de escuelas y colegios fue el repertorio más común de estos actores.

De igual manera, las vecinas y los vecinos, tercer actor con mayor registro de protestas en el período $(10,2 \%)$, recurrieron a repertorios de protesta mayoritariamente noinstitucionales $(62,8 \%)$ (gráfico 6), de las cuales se destaca el bloqueo $(35,6 \%)$ y la concentración $(20,0 \%)$ como las medidas de presión más comunes para posicionar sus demandas. 


\section{GRÁFICO 6 \\ ACCIONES DE PROTESTA POR PARTE DE LAS VECINAS Y LOS VECINOS \\ SEGÚN CAMPO DE ACCIÓN \\ COSTA RICA, 2014-2018 \\ (DISTRIBUCIONES PORCENTUALES)}

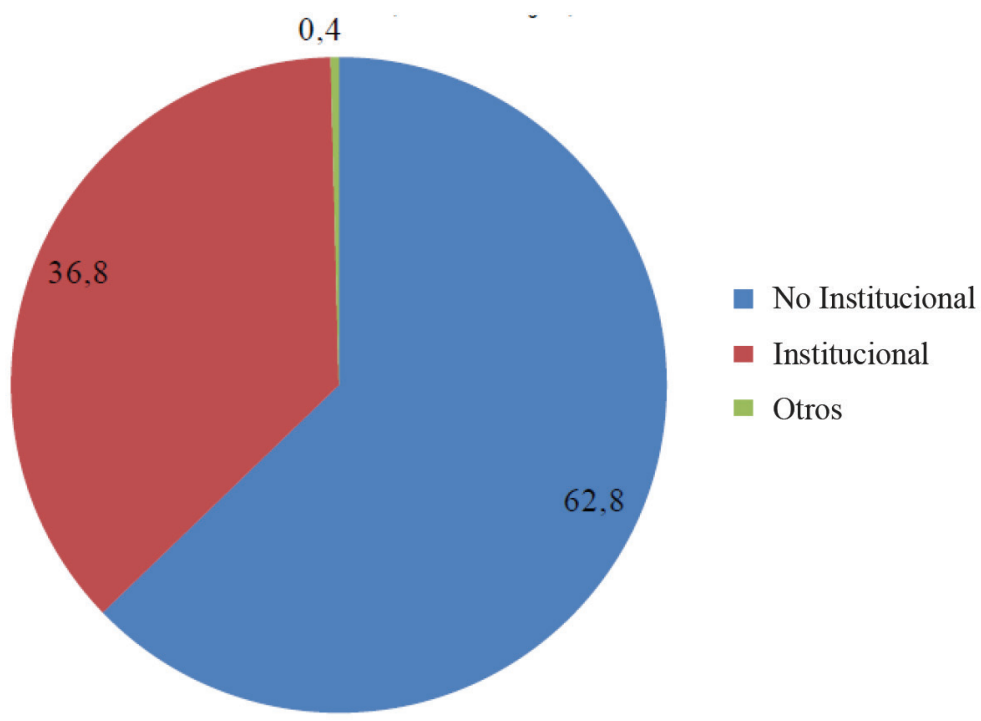

Fuente: $\quad$ PROTESTAS, base de datos colectivas, IIS-UCR, 2014-2018.

Las principales demandas de las vecinas y los vecinos se relacionan con dinámicas locales y comunitarias, entre las que destacan el acceso a agua potable, el mejoramiento de rutas $y$ la defensa del medio ambiente, todas estas inscritas en escenarios locales y con escasa articulación entre los eventos de protesta.

En síntesis, durante el cuatrienio de gobierno de Solís se muestra un decrecimiento sostenido en la cantidad de protestas registradas, tanto para el total de los datos como en los registros de las acciones de protesta de la población trabajadora y de los vecinos y las vecinas. Asimismo, para el conjunto de registros de la base de datos, se denota una predominancia de los formatos institucionales de protesta, los cuales fueron recurridos fundamentalmente por actores más organizados y con más recursos como el sector trabajador, mediante los sindicatos. Por su parte, actores locales menos organizados y con menos recursos políticos, tales como estudiantes, madres y padres, recurrieron a formatos no-institucionales.

Este fenómeno es explicado por distintos teóricos de los movimientos sociales, como Tarrow (1997), quien indica que la protesta contenciosa es, en muchas ocasiones, el único recurso con el que cuentan ciertos actores para incidir en el espacio de la política institucional frente a otros actores con más y mejores recursos. De esta manera, las rutinas y las estrategias de acción de los actores demandantes, las cuales se pueden precisar mediante la reconstrucción de sus repertorios de protesta, ofrecen una entrada para entender el tipo de relaciones que se construyen entre los actores de la contienda política, pues la mayor o menor presencia de repertorios no-institucionales sugiere una profundización de la conflictualidad social y de la producción de antagonismos en una determinada coyuntura.

Considerando lo anterior, en las siguientes secciones se propone una entrada teórica y 
una hipótesis de trabajo a futuro para tratar de entender por qué decreció la protesta durante el gobierno de Solís.

\section{LA ESTRUCTURA DE OPORTUNIDADES POLÍTICAS (EOP) Y LA DINÁMICA DE LA PROTESTA}

Entre la teoría de los movimientos sociales, hay todo un corpus teórico dedicado a entender la dinámica que se establece entre la llamada política institucional y la política de la calle (o noinstitucional). Diferentes autores han planteado que las características del entorno político en el que se inscriben las protestas sociales son determinantes para entender el carácter de estas, sus posibilidades de desarrollo y los resultados que se obtienen de sumarse a este tipo de acciones.

Particularmente, autores pertenecientes a las corrientes estadounidenses de estudios sobre los movimientos sociales, tales como Tarrow (1997 y 1999) y McAdam (1999), han postulado que la Estructura de Oportunidades Políticas (EOP), entendida como "señales continuas, aunque no necesariamente permanentes, formales o nacionales, [del entorno político] que son percibidas por los actores sociales y que les alientan o desalientan a usar los recursos con los que cuentan..." (Tarrow, 1999, p. 89), constituye el factor determinante para explicar la dinámica de la protesta social.

Según este planteamiento, el tipo de oportunidades políticas presentes en el entorno donde se desarrollan los movimientos sociales condicionarán no solo su formación, sino su desarrollo, resultados y rutinas de acción. Una EOP predominantemente abierta ofrece mayores condiciones políticas para la protesta social $y$, particularmente, para que los actores consigan establecer espacios de interlocución con sus contrincantes. Por el contrario, una EOP predominantemente cerrada, no necesariamente elimina la protesta, pero sí plantea una relación distinta entre los actores de la contienda política, en tanto incrementa el costo de protestar (Tarrow, 1997 y Favela, 2012) $)^{12}$.

12 Es necesario acentuar que se hace referencia a características predominantes de los sistemas políticos, pues la mayoría de estos presentan
Dentro de las dimensiones de análisis que configuran la EOP, se destaca el grado de acceso institucional con el que cuentan los actores de la protesta, poniendo énfasis en los mecanismos institucionales que se ofrecen para que dichos actores puedan plantear $y$ procesar sus demandas a la institucionalidad.

De esta manera, las oportunidades políticas no solo influyen en el cuándo se forma la acción colectiva, sino en el cómo, es decir, en las estrategias y rutinas de acción (McAdam, McCarthy y Zald, 1999). La forma que toma la acción colectiva, los repertorios a los que recurran los actores demandantes y el tipo de relaciones que se generan entre estos y sus contrincantes, mantiene una íntima relación con las oportunidades políticas presentes.

Por ejemplo, un actor local con escaso acceso a las instituciones donde se toman las decisiones, probablemente tendrá que hacer uso de rutinas no-institucionales con un carácter marcadamente transgresor, como es el caso de los bloqueos de calles, ante la necesidad de llamar la atención de los actores demandados. Por el contrario, ciertos actores, sobre todo los que cuentan con mayores recursos políticos, económicos y organizativos, como es el caso de los sindicatos, pueden conseguir colocar sus demandas en la institucionalidad, recurriendo a rutinas institucionales, tales como, las declaraciones públicas o las denuncias ante entidades ${ }^{13}$.

diferentes combinaciones de oportunidades políticas abiertas y cerradas. De hecho, la mayoría de autores coincide en señalar que es precisamente en sistemas donde se presentan elementos abiertos y cerrados donde se hace más usual que se desarrolle la protesta social.

13 En este punto, es fundamental recuperar lo señalado por Tarrow (1997 y 1999) en referencia a que las oportunidades políticas no son necesariamente permanentes, formales o nacionales, lo cual marca también su carácter cambiante. Precisamente por esto encuentra sentido que los diferentes actores demandantes desarrollen distintos tipos de relaciones con un mismo actor demandado, por ejemplo, el gobierno; el cual mediante sus distintos mecanismos y entramados institucionales define su propia estrategia de interacción y respuesta según los actores que protestan. Como consecuencia, las oportunidades políticas son diferenciadas según 
Ahora bien, para autores como Tarrow (1997 y 1999), son las oportunidades políticas coyunturales (por oposición a las llamadas estructurales), entre las que se incluye la mencionada de acceso a la institucionalidad, las que más pesan para explicar la dinámica de la protesta social. Los cambios coyunturales en las oportunidades políticas ofrecen condiciones para que los actores de la protesta desarrollen sus acciones e incidan en diferentes grados en el carácter de dichas acciones. Este tipo de cambios pueden estar marcados por factores, tales como, el cambio de gobierno $y$ de las formas de manejo de la protesta que influyen en la dinámica que se establece entre los actores de la contienda política (McAdam, Tarrow y Tilly, 2005).

Precisamente en esta dirección se propone como hipótesis de trabajo, para explorar en el futuro, que la caída en el número de acciones de protesta y la tendencia general identificada de predominancia de los repertorios institucionales sobre los no-institucionales, podría relacionarse con el cambio de gobierno, es decir, la llegada de Luis Guillermo Solís a la presidencia de la República, $y$, particularmente, a la forma de manejo de la protesta que dicho gobierno propuso.

\section{¿UNA ESTRUCTURA DE OPORTUNIDADES POLÍTICAS ABIERTAS EN EL GOBIERNO DE SOLÍS?: UNA HIPÓTESIS DE TRABAJO A FUTURO}

Tal como se señaló anteriormente, las expectativas de cambio generadas por el triunfo electoral de Luis Guillermo Solís, pronto se desinflaron ante una serie de acontecimientos que desembocaron en un distanciamiento entre la población (que lo había apoyado mayoritariamente en la segunda ronda electoral de abril de 2014) y el gobierno. Pese a esto, los datos referidos a las acciones de protesta registradas durante el cuatrienio, sugieren que frente a dicho distanciamiento, un sector considerable de la población no tomó la ruta de las calles para manifestar su descontento. En este sentido,

el actor que protesta, incidiendo en las rutinas de acción y en la dinámica de la conflictualidad social y política en general. resulta interesante plantearse hipótesis que contribuyan a explicar por qué se redujo la protesta durante el gobierno de Solís.

En el caso de este artículo, se propone como hipótesis de trabajo a futuro que el triunfo electoral y posterior gobierno de Solís, generó un entorno político para los actores de la protesta, marcado por un mayor grado de acceso a las instituciones $y$ por un estilo de manejo de la protesta con un uso predominante de mecanismos institucionales, que desembocó en una mayor institucionalización de la protesta $y$ de ciertos actores demandantes.

En un estudio, Guillén (2016) había propuesto una ruta explicativa similar para entender la reducción de las acciones de protesta durante los primeros dos años de gobierno de Solís. Incluso, la autora recurrió a entrevistas con dirigentes sindicales y con autoridades del MTss para probar que las oportunidades políticas abiertas por el gobierno habían ocasionado la baja de la protesta. En este trabajo se contrastan los datos de la protesta registrados para los últimos dos años de gobierno de Laura Chinchilla (2010-2014) con los primeros dos de Solís (2014-2015), lo que constata la reducción de la protesta en el período intergubernamental. Mientras, los dos últimos años de Chinchilla se caracterizaron por altos niveles de protesta (Guillén, 2016), los primeros dos de Solís, mostraron una tendencia contraria, la cual continuó durante sus últimos dos años (Alvarado y Martínez, 2018).

Partiendo de este hecho, una primera hipótesis para explicar la reducción de la protesta podría plantearse en el sentido de una disminución de la conflictualidad social en general, lo cual se tradujo en menos protestas. No obstante, esta hipótesis pareciera poco plausible pues durante el gobierno de Solís siguieron presentes los principales nudos de conflicto registrados en el gobierno de Chinchilla, a los cuales se sumaron otros ${ }^{14}$.

14 Durante los gobiernos de Óscar Arias (2006-2010) y Laura Chinchilla (2010-2014) se registraron importantes nudos de conflictualidad social, entre los que destacan la campaña de protesta contra el TLC, las luchas antimineras como la de Crucitas, las antipiñeras y los conflictos por la concesión 
En este sentido, pareciera que el cambio que podría explicar la merma en la cantidad de acciones de protesta durante el gobierno de Solís no debe buscarse en la reducción de las tensiones sociales, sino en el estilo de manejo de la protesta que cada gobierno desarrolló y en el tipo de relaciones que se fueron construyendo entre los actores de la contienda política (Guillén, 2016), lo cual se tradujo en más y mejores oportunidades políticas para ciertos actores demandantes, específicamente aquellos que contaron con ciertos recursos políticos para colocar sus demandas en la institucionalidad sin la necesidad de recurrir predominantemente a la protesta callejera, como fue el caso de las organizaciones sindicalistas, las LGBTI o las ambientalistas.

Guillén (2016) plantea dos factores determinantes, en términos de las oportunidades políticas habilitadas por el gobierno de Solís, particularmente en referencia a su relación con el actor sindical. En primer lugar, señala que entre el gobierno y los sindicatos había una relación más cercana, debido a que el PAC había estado implicado en diferentes luchas sociales desde su fundación en la primera década del 2000.

En el caso de los sindicatos, sobre todo los del sector público, una señal de que el camino institucional parecía "abrirse", fue el apoyo de Solís a la Reforma Procesal Laboral (RPL), la cual era una demanda de las organizaciones sociales $y$, particularmente,

de obra pública en el caso de la Terminal de Contenedores de Moín (тсM). Estas fueron luchas que iniciaron o se intensificaron en el gobierno de Arias. En el de Chinchilla se sumaron otras como el conflicto por la concesión de la carretera San José-San Ramón o las luchas de las mujeres $y$ de las poblaciones LGBTI. Igualmente, se dieron conflicto en torno a la sostenibilidad de regímenes de pensiones como el de la Caja Costarricense de Seguro Social (ccss). Por su parte, en el gobierno de Solís persistieron nudos originados en los gobiernos anteriores, tales como el de la construcción de la TCM, las luchas de las mujeres y de las poblaciones LGBTI, la situación de la ccss, entre otros. A estos se sumaron conflictos con los taxistas ocasionados por la entrada en funcionamiento de Uber o la implementación de los programas de educación sexual en el MEP. sindicales, que había sido vetada por la presidenta Chinchilla.

En segundo lugar, relacionado con lo primero, la decisión de Solís de colocar a figuras cercanas a las organizaciones sociales en puestos de mando, propició condiciones para el diálogo con los actores no-institucionales, pues, hasta cierto punto, habilitó un "lenguaje común" entre los actores de la contienda política. Entre otros casos, se pueden mencionar los de Ana Helena Chacón en la vicepresidencia de la República ${ }^{15}$, Harold Villegas en el MTss o Yamileth Astorga en el AyA.

Sumado a lo anterior, desde su inicio, el gobierno de Solís recurrió a la conformación de mesas de diálogo con la finalidad de institucionalizar la interacción entre el ejecutivo $y$ los actores no-institucionales ${ }^{16}$. Si bien, en este trabajo no es posible determinar la eficacia de dichas mesas de diálogo, sí se considera un factor a considerar como parte de la hipótesis planteada. El recurso a dialogar fue un mecanismo constantemente habilitado ante el incremento de tensiones con diferentes actores, tales como, los taxistas ${ }^{17}$, las poblaciones indígenas ${ }^{18}$ y las campesinas.

15 Desde su puesto como vicepresidenta, Chacón se transformó en una aliada estratégica de la población LGBTIQ+, impulsando acciones en apoyo a la lucha de este sector. La posición que tomó el gobierno de Solís frente a las demandas de esta población, generó un entorno político más abierto que se tradujo en un cambio en la estrategia de las organizaciones LGBTIQ+, las cuales prácticamente dejaron de lado la protesta callejera para centrarse en el diálogo con el gobierno.

16 Con base en una primera consulta de comunicados oficiales emitidos por el gobierno de Costa Rica entre 2014 y 2018, se identificaron un total de 12 procesos de diálogo con distintos actores sociales, entre los que destacan transportistas, poblaciones indígenas, campesinas, costeras, entre otras.

17 Fue precisamente en el segundo año de gobierno de Solís que iniciaron las protestas de este sector contra la entrada en funcionamiento de la empresa Uber en Costa Rica.

18 El gobierno de Solís debió enfrentar uno de los conflictos indígenas más importantes de las últimas décadas en el país. En 2010, tras una acción de desalojo contra un grupo de dirigentes indígenas que permanecía protestando en la sede 
En esta dirección, se considera que los elementos mencionados pudieron resultar determinantes en la dinámica entre los actores de la contienda política, pues permitieron que ciertos actores de la protesta, sobre todo, los que contaban con más recursos políticos y organizaciones formales con mayores niveles de consolidación, recurrieran predominantemente a los mecanismos institucionales y espacios formales para procesar sus demandas, ante una lectura que indicaba un entorno político más abierto y la presencia de potenciales aliados entre el poder ejecutivo. Por el contrario, para otros actores, particularmente los de carácter local, con menos recursos y organizaciones formales, el entorno fue similar a lo registrado en gobiernos anteriores ${ }^{19}$.

En resumen, el conjunto de elementos resaltados en esta sección, parecieran agregar a la hipótesis de que la reducción de la protesta social durante el gobierno de Solís obedeció, principalmente, a las oportunidades políticas abiertas por el ejecutivo a una serie de actores demandantes, permitiéndoles colocar e institucionalizar sus demandas. Esto redundó en que actores sociales organizados que permanecieron constantemente en la calle durante los dos gobiernos anteriores, se encontraran con un entorno político más abierto, generando que estos destinaran la mayoría de sus recursos políticos a la gestión de sus demandas mediante la institucionalidad, cambiando la dinámica de la contienda política. No obstante, confirmar o no

legislativa, un sector de indígenas de la comunidad de Salitre, en el Pacífico Sur costarricense, decidió iniciar un proceso de recuperación de tierras mediante el recurso a la acción directa, ocasionando enfrentamientos entre la población indígena recuperante $y$ la población no-indígena que mantenía posesiones ilegales de tierra en el territorio indígena. El gobierno de Solís reaccionó a este conflicto, redirigiendo buena parte de los recursos del Viceministerio de la Presidencia para gestionar las tensiones entre indígenas y no-indígenas.

19 Esto último se refleja parcialmente en las diferencias retratadas en este trabajo entre los niveles de protesta $y$ las diferencias en el uso de repertorios institucionales y no-institucionales, identificadas para los principales actores demandantes de la coyuntura. dicha hipótesis, trasciende los objetivos de este trabajo, por lo que la intención ha sido la de posicionar una serie de elementos a considerar en investigaciones futuras.

\section{CONCLUSIONES}

El presente artículo buscó contribuir a la discusión sobre la dinámica de la contienda política en Costa Rica, mediante el monitoreo sistemático de las acciones de protesta registradas en la prensa escrita durante el cuatrienio de gobierno de Luis Guillermo Solís (2014-2018).

Siguiendo la metodología de AEP, fue posible registrar un total de 2475 acciones de protesta durante el período. Asimismo, se constató la tendencia a la reducción sostenida de los niveles de protesta $y$ la predominancia de repertorios institucionales sobre los no-institucionales.

La declaración pública, las concentraciones, las reuniones y los bloqueos fueron los repertorios de uso común, mientras que los repertorios clásicos como el paro o la huelga fueron escasamente puestos de acto.

Los tres actores demandantes más importantes fueron: la población trabajadora; las madres, los padres y el estudiantado; las vecinas y los vecinos; destacando particularmente los primeros, quienes fueron protagonistas en prácticamente 1 de cada 2 acciones de protesta en el período.

Entre la población trabajadora, los repertorios institucionales fueron los más comunes. Mientras que en los otros dos actores, fueron los no-institucionales, con un predominio de los cierres (bloqueos) de los centros de educación, tanto a nivel de primaria como de secundaria.

Por otra parte, para dar una explicación a la reducción sistemática de la cantidad de protestas durante el cuatrienio, se propuso una hipótesis de trabajo a futuro, según la cual, las oportunidades políticas abiertas por el gobierno de Solís pudieron determinar la reducción de los niveles de protesta, debido a la combinación de una serie de factores entre los que se incluyen: un mayor acceso a las instituciones, una mayor cercanía entre los actores institucionales $y$ los no-institucionales, $y$ el recurso a las mesas de diálogo. No obstante, 
la comprobación de dicha hipótesis escapa a los objetivos de este estudio.

Por último, este artículo, resultado de un proceso sistemático de monitoreo de la protesta social en el país, constituye un insumo académico para la comprensión de la conflictualidad social en Costa Rica. En esta medida, se presenta la base de datos PROTESTAS como un recurso que permite registrar tendencias de la conflictualidad social nacional e identificar patrones, continuidades $y$ discontinuidades en términos de los repertorios de protesta, los actores demandantes, las demandas y los actores demandados, entre otros, a escala nacional, regional y local. De esta manera, persigue como objetivo fundamental reconstruir el panorama general de la protesta social en el país, lo cual constituye un rico insumo para entender las relaciones entre esta y los procesos sociales, políticos y económicos.

\section{REFERENCIAS}

Alvarado, A. y Martínez, G. (2018). La protesta social en el gobierno de Luis Guillermo Solís (2014-2018). Inédito.

Álvarez, L. (2011). El mito democrático costarricense. La constitución de la práctica política en períodos de conflicto social. Ciudad de México: Flacso.

Álvarez, L. (2015). El mito democrático costarricense y la invisibilización del conflicto. En: Vázquez, L.D. (coord.). De la democracia liberal a la soberanía popular. Vol. 1. Argentina: CLACSO.

Cadena-Roa, J. (2016). Las organizaciones de los movimientos sociales y los movimientos sociales en México, 2000-2014. Ciudad de México: Friedrich Ebert Stiftung.

Calderón, F. (2012). La protesta social en América Latina. Buenos Aires: Siglo XxI Editores.

Cascante, M.J. y Pignataro, A. (2018). Los electorados de la democracia costarricense: percepciones ciudadanas y participación en torno a las elecciones nacionales de 2014. San José: IFED-TSE.

Centro de Investigación y Estudios Políticos. (2014). Estudios de Opinión
Sociopolítica. Informe de la Encuesta de Enero. San José: CIEP-UCR. Recuperado de https://ciep.ucr.ac.cr/publicaciones/informe-de-opini\%c3\%B3n-p\%C3\%BAblicaencuestas-2014

Centro de Investigación y Estudios Políticos. (2015). Informe Encuesta Sociopolítica Noviembre 2014. San José: CIEP-UCR. Recuperado de https://ciep.ucr.ac.cr/ sites/default/files/Informe-encuesta-CIEPnoviembre-2014.pdf

Centro de Investigación y Estudios Políticos. (2016). Informe de Resultados de la la Encuesta de Opinión Sociopolítica Agosto 2016. San José: CIEP-UCR. Recuperado de https://ciep.ucr.ac.cr/ sites/default/files/Informe-encuesta-deagosto-2016-CIEP.pdf

Centro de Investigación y Estudios Políticos. (2018). Informe de Resultados de la Encuesta de Opinión Sociopolítica Agosto 2018. San José: CIEP-UCR. Recuperado de https://ciep.ucr.ac.cr/ sites/default/files/Informe-encuestaagosto22-2018.pdf

Favela, D. (enero-abril, 2002). La estructura de oportunidades políticas de los movimientos sociales en sistemas políticos cerrados: examen del caso mexicano. Estudios Sociológicos, 20 (58), 91-121.

Guillén, M.J. (2016). El panorama de las acciones colectivas en Costa Rica. Ponencia preparada para el XII Informe Estado de la Nación en Desarrollo Humano Sostenible.

McAdam, D.; McCarthy, J. y Zald, M. (1999). Oportunidades, estructuras de movilización y procesos enmarcadores: hacia una perspectiva sintética y comparada de los movimientos sociales. En McAdam, D.; McCarthy, J. y Zald, M. (eds.). Movimientos sociales: perspectivas comparadas. Madrid: Istmo.

McAdam, D. (1999). Orígenes terminológicos, problemas actuales, futuras líneas de investigación. En McAdam, D.; McCarthy, J. y Zald, M. (eds.). Movimientos sociales: perspectivas comparadas. Madrid: Istmo. 
McAdam, D.; Tarrow, S. y Tilly, C. (2005). Dinámica de la contienda política. Barcelona: Editorial Hacer.

Raventós, C.; Fournier, M.; Ramírez, O.; Gutiérrez, A. y García, J. (2005). Abstencionistas en Costa Rica: ¿quiénes son y por qué no votan? San José: EUCR, IIDHCAPEL $y$ TSE.

Raventós, C.; Fournier, M.; Fernández, D. y Alfaro, R. (2012). Respuestas ciudadanas ante el malestar con la política: salida, voz y lealtad. San José: UCR y TSE.

Retamozo, M. y Trujillo, L. (julio-diciembre, 2018). Cambios estructurales y prácticas de movilización política en Argentina. Dos ciclos políticos en perspectiva (1989-2002 y 2003-2015). Papel Político, 23 (2), 1-19.

Schuster, F.; Pérez, G.; Pereira, S.; Armesto, M.; Armelino, M.; García, A.; Natalucci, A.; Vázquez, M. y Zipcioglu, P. (2006). Transformaciones de la protesta social en Argentina. Documento de Trabajo 48. Argentina: Instituto de Investigaciones Gino Germani.
Svampa, M. (mayo de 2009). Protesta, movimientos sociales y dimensiones de la acción colectiva en América Latina. Ponencia preparada para las "Jornadas de Homenaje a Charles Tilly". Madrid: Universidad Complutense-Fundación Carolina.

Tarrow, S. (1997). El poder en movimiento. Los movimientos sociales, la acción colectiva y la política. Madrid: Alianza Editorial.

Tarrow, S. (1999). Estado y oportunidades: la estructuración política de los movimientos sociales. En McAdam, D.; McCarthy, J. y Zald, M. (eds.). Movimientos sociales: perspectivas comparadas. Madrid: Istmo.

Tilly, C. (2002). Repertorios de acción contestataria en Gran Bretaña: 1758-1834. En Traugott, M. (comp.). Protesta social, repertorios y ciclos de la acción colectiva. Barcelona: Editorial Hacer.

Fecha de ingreso: 08/08/2019 Fecha de aprobación: 17/10/2019 